\title{
ANTIBIOGRAM STUDY AND ANTIBIOTIC USE EVALUATION USING GYSSEN METHOD IN PATIENTS WITH DIABETIC FOOT
}

\author{
Umul Fadlilah $^{1}$, Didik Hasmono ${ }^{1}$, Yudi Agung Wibisono ${ }^{2}$, Mia Melinda ${ }^{2}$ \\ ${ }^{1}$ Department of Clinical Pharmacy, Faculty of Pharmacy, Universitas Airlangga \\ ${ }^{2}$ Department of Internal Medicine, Mardi Waluyo Hospital, Blitar
}

\begin{abstract}
ABSTRAK
Latar belakang. Infeksi kaki adalah masalah umum dan serius pada orang dengan diabetes, yang memerlukan pengelolaan tepat (pendekatan diagnostik dan terapetik) agar dapat disembuhkan. Regimen antibiotika empiris harus didasarkan pada data klinis dan pola kuman yang tersedia, tetapi terapi definitif harus didasarkan pada hasil kultur jaringan yang terinfeksi. Kesulitan untuk pemilihan antibiotika pada terapi awal dan penggunaan yang kurang bijak menjadi masalah tersendiri dan beresiko pada munculnya resistensi antibiotika. Perlu adanya evaluasi penggunaan antibiotika untuk mendorong penggunaan yang lebih bijak. Tujuan. Menganalisis pola kuman pada kaki diabetik dan uji sensitifitasnya terhadap antibiotika, menganalisis antibiotika empiris yang dapat direkomendasikan, dan menganalisis penggunaan antibiotika dengan metode Gyssen. Metode. Penelitian ini adalah studi analisis observasional (deskriptif non-eksperimental), retrospektif dan prospektif pada pasien infeksi kaki diabetik yang memenuhi kriteria inklusi. Data tetrospektif digunakan untuk mengenalisis pola kuman dan uji sensitifitas terhadap antibiotika dan data prospektif digunakan untuk mengevaluasi penggunaan antibiotika berdasarkan pola kuman yang ada, selama periode akhir Maretawal Agustus 2015 di RSUD Mardi Waluyo Kota Blitar. Evaluasi dilakukan dengan metode Gyssen. Hasil, sampel data retrospektif diperoleh 30 temuan kuman infecting selama bulan Agustus 2014-Maret 2015. Prevalensi kuman gram negatif sebanyak 53,33\% dengan jenis kuman terbanyak E.coli dan Klebsiella oxytoca (13,33\%), dan gram positif sebanyak 46,67\% dengan kuman terbanyak Staphylococcus spp. dan Streptococcus spp. Dari data prospektif yang memenuhi kriteria inklusi sebanyak 13 pasien dengan prevalensi kuman terbanyak gram negatif adalah Klebsiella oxytoca (28,57\%), dan terbanyak gram positif adalah Staphylococcus auerus $(35,71 \%)$. Sementara analisis kualitatif penggunaan antibiotika dilakukan terhadap 50 jenis pemberian antibiotika. Hasil analisis kualitatif menggunakan metode Gyssens didapatkan penggunaan kategori sebanyak $62 \%$, penggunaan kategori sebanyak $2 \%$, kategori sebanyak 14\%; kategori 2 B sebanyak 26\%; kategori $3 A$ sebanyak 10\%; kategori 4 A sebanyak $52 \%$, kategori $4 B$ sebanyak 6\%; kategori 4 C sebanyak $8 \%$ dan tidak ada penggunaan antibiotika yang masuk kategori V dan kategori VI. Simpulan. Dari analisis gyssen ini dapat diperoleh data bahwa penggunaan antibiotika pada pasien kaki diabetik di RSUD Maerdi Waluyo Kota Blitar didominasi oleh ketidak tepatan dalam pemilihan antibiotika, dan ketidaktepatan dalam interval pemberian antibiotika. (FMI 2016;52:198-208)
\end{abstract}

Kata kunci: pola kuman, antibiotik, infeksi kaki diabetik, metode Gyssen

\begin{abstract}
Foot infection is a common and serious problem in people with diabetes, which require proper management (diagnostic and therapeutic approaches) that can be cured. Empiric antibiotic regimen should be based on clinical data and bacteria pattern that are available, but definitive therapy should be based on the results of the infected tissue culture. The selection of initial antibiotic therapy was difficult and unwise use can lead to antibiotic-resistant. Evaluation is needed for using antibiotics to benefit wisely. The aim of this research is to analyzed the pattern of bacteria in diabetic foot and to its sensitivity test to antibiotics, analyze empiric antibiotics that can be recommended, and analyzed the use of antibiotics by Gyssen method. Data was analyzed with observational studies (descriptive non-experimental), retrospectively and prospectively in patients diabetic foot infection that met inclusion criteria. Retrospective data are used to analyzed bacteria pattern and its sensitivity test, while prospective data are used to evaluated the use of antibiotics based on bacteria pattern, during the period of late March-early August 2015 at Mardi Waluyo Hospital. Evaluation was conducted by Gyssen method. The results, retrospective data samples obtained 30 infection bacteria during August 2014-March 2015. The prevalence of gram-negative bacteria as $53.33 \%$ with most types of bacteria E.coli and Klebsiella oxytoca (13.33\%), and gram-positive bacteria as $46.67 \%$ with the highest bacteria are Staphylococcus spp. and Streptococcus spp. From the prospective data in inclusion criteria, 13 patients with the highest prevalence of gram-negative bacteria are Klebsiella oxytoca (28.57\%), and most gram-positive Staphylococcus auerus (35.71\%). While the qualitative analysis of antibiotic use was conducted on 50 types of antibiotics. The results of the qualitative analysis using Gyssens method obtained category as $62 \%, 2 \%, 14 \%, 2 B$ category as $26 \%$, $3 \mathrm{~A}$ category as $10 \%, 4 \mathrm{~A}$ category $52 \%, 4 \mathrm{~B}$ category as $6 \%, 4 \mathrm{C}$ category as $8 \%$ and there are no use of antibiotics in the category $\mathrm{V}$ and VI. Conclusions, Gyessen method can show that the use of antibiotics in diabetic foot patients in Mardi Waluyo hospital is dominated by inaccuracy in choice of antibiotic, and inaccuracies in the interval antibiotics. (FMI 2016;52:198-208)
\end{abstract}

Keywords: bacteria patterns, antibiotics, diabetic foot infections, Gyssens method

Correspondence: Umul Fadlilah, Department of Clinical Pharmacy, Faculty of Pharmacy, Universitas Airlangga, Jalan Dharmawangsa Dalam, Surabaya 60286. 


\section{INTRODUCTION}

Foot infection is a common and serious problem in people with diabetes. Diabetic foot infections or Diabetic Foot Infection (DFI) usually starts with injuries, most often neuropathic ulceration. While all wounds are colonies of microorganisms, the presence of infection is defined by the findings of inflammation or pus. Infections and classified into mild (superficial and limited in size and depth), moderate (deeper or wider), or severe (accompanied by signs of systemic or metabolic disorders). This classification system, along with vascular assessment, help determine which patients need to be hospitalized, which may require special imaging procedure or surgical intervention, and requiring amputation (Lipsky 2012).

The prevalence of diabetic ulcers in Indonesia amounted to $15 \%$ of patients with DM. Most diabetes care is always associated with diabetic ulcers. Mortality and amputation remains high, and the fate of postamputation in diabetic patient is still very bad, as many as $14.3 \%$ will die within a year of post-amputation and $37 \%$ will die of 3 years post-amputation (Waspadji 2006). Meanwhile, according to Riyanto, amputation figure reached $30 \%, 32 \%$ mortality rate, and diabetic ulcers is because the majority of hospital treatment by $80 \%$ in the case of diabetes mellitus (Riyanto 2007).

Mardi Waluyo Hospital Kota Blitar, of Profile Installation Medical Record of 2013 data showed that cases of hospitalization for diabetes mellitus ranks third after a stroke and a heart big, that some 495 cases or $3.51 \%$ of all cases of hospitalization. Of that number 66 cases $(13.33 \%)$ were diabetic foot infections. In 2014 increased to 524 cases (3.8\%), ranks second after stroke. $82(15.65 \%)$ cases were DM patients with diabetic foot infections. From these data seen an increase in the number of cases. While the treatment of patients with diabetic foot infections the use of antibiotics has been no recommendation related to the pattern of the infecting organism (Profil Mardi Waluyo Hospital Medical Records Kota Blitar 2013-2014).

Most diabetic foot infections are polymicrobial, with gram-positive cocci, especially staphylococci which is the most common causative organism. Gram-negative rods often copathogen in chronic infection or the antibiotic treatment, and obligate anaerobes may copathogen on ischemic or necrotic wounds (Frier 2006). Wound infection without evidence of soft tissue or bone does not require antibiotic therapy. Empirical antibiotic therapy can be targeted narrowly at GPC (gram positive cocci) in many patients with acute infection, but those who are at risk for infection or chronic antibiotic resistant organisms, or severe infection usually requires a broader spectrum of drugs (Lipsky 2012).

Evaluation of the use of antibiotics in general can be performed quantitatively and qualitatively. To evaluate the quality of antibiotics there are many parameters that are used as dose accuracy, precision interval of administration, route of administration, and others. Qualitative assessment allows us to know whether antibiotics were given was appropriate, conducted by in-depth analysis of the medical records, also known as practical audit. Qualitative assessment is rarely done because of the lack of standardization, the methodology is difficult, and requires human resources (Cusini 2010). Nevertheless qualitative discussion of antibiotics can encourage clinicians to be more prudent in the use of antibiotics. Gyssen groove is one of the algorithms used for the qualitative evaluation of the use of antibiotics (Habib 2014).

During Mardi Waluyo Hospital in Kota Blitar have never done research on patterns of bacteria in patients with diabetic foot and there is no information related to empiric antibiotics based on the pattern of germs. Therefore, with this study are expected to be obtained from the data pattern of the bacteria on a culture of microbiological sample of pus patients with diabetic foot and sensitivity test antibiotics against germs (retrospective data), which later became the basis of the selection of empiric antibiotics, and then carried out qualitative evaluation of the use of antibiotics prospectively using Gyssens groove. Evaluation of the use of antibiotics in general can be performed quantitatively and qualitatively. To evaluate the quality of antibiotics there are many parameters that are used as dose accuracy, precision interval of administration, route of administration, and others. Qualitative assessment allows us to know whether antibiotics were given was appropriate, conducted by in-depth analysis of the medical records, also known as practical audit. Qualitative assessment is rarely done because of the lack of standardization, the methodology is difficult, and requires human resources (Cusini 2010). Nevertheless qualitative discussion of antibiotics can encourage clinicians to be more prudent in the use of antibiotics. Gyssen groove is one of the algorithms used for the qualitative evaluation of the use of antibiotics (Habib 2014).

During Mardi Waluyo Hospital in Kota Blitar have never done research on patterns of bacteria in patients with diabetic foot and there is no information related to empiric antibiotics based on the pattern of germs. Therefore, with this study are expected to be obtained from the data pattern of the bacteria on a culture of microbiological sample of pus patients with diabetic 
foot and sensitivity test antibiotics against germs (retrospective data), which later became the basis of the selection of empiric antibiotics, and then carried out qualitative evaluation of the use of antibiotics prospectvely using Gyssens groove.

\section{MATERIALS AND METHODS}

Making antibiogram done by connecting the data bacteria culture results and antibiotic sensitivity test in the form of a table. Based on antibiogram can be seen the percentage of antibiotic sensitivity and categories that can be recommended for empirical use. Descriptive analysis is used to assess the results of the evaluation of the quality of the use of antibiotics with Gyssen method, first, time use of antibiotics (category I); Second, the regimentation dose, interval, these antibiotics (category II A - II C); Thirdly, the duration of use of antibiotics (category III A - III B); Fourth, the choice of the form of clinical efficacy, toxicity, price and sprektrum antibiotic coverage (category IV A - IV D); and fifth, an indication of the use of antibiotics (category V - VI), which will then be given a percentage on each key subject of evaluation.

\section{Analysis}

The analysis is conducted qualitatively by methods Gyssen against the use of antibiotics by referring to the data patterns of bacteria and sensitivity to antibiotics.

\section{RESULTS}

\section{Data Retrospective}

From skitar 42 patients hospitalized with a diagnosis of diabetic foot infection in hospitals Mardi Waluyo there were 23 patients who met the inclusion criteria and pus obtained 28 cultures, 30 isolates of bacteria and 20 types of germs infecting.

\section{Germs Profile in Patients with Diabetic Foot}

Table 1 shows the types of bacteria that cause infections in the diabetic foot hospital inpatient Mardi Waluyo period August 2014 - March 2015. Of the 23 patients obtained 28 results hulkur pus which produces 30 isolates of bacteria with prevalence of gram-negative bacteria as much as $53.33 \%$ and gram-positive in $46.67 \%$.

\section{Sensitivity Profile Germs on the Diabetic Foot Infection Antibiotics}

Table 2 displays the results of the research profile pussy isolates, the prevalence and antibiotic sensitivity test results. Percentage (\%) of antibiotic sensitivity is obtained by calculating the number of antibiotic-sensitive test divided by the number of isolates germs multiplied by $100 \%$.

Table 1. Type of germs that cause diabetic foot infection period from August to March, 2015

\begin{tabular}{|c|c|c|c|c|c|}
\hline No & Type of Germs & $\mathrm{N}(\%)$ & Gram + / - & $\%$ Gram $-/+$ & Note \\
\hline 1 & Escherichia coli & $4(13,33)$ & - & $53.33 \%$ & \\
\hline 2 & Klebsiella oxsytoca & $3(10,0)$ & - & & Klebsiella spp (13.33\%) \\
\hline 3 & Klebsiella pneumoniae & $1(3.33)$ & - & & \\
\hline 4 & Citrobacter freundii & $2(6,67)$ & - & & \\
\hline 5 & Enterobacter sakazakii & $1(3.33)$ & - & & Enterobacter spp (6.67\%) \\
\hline 6 & Enterobacter agglomerans & $1(3.33)$ & - & & \\
\hline 7 & Pseudomonas aeruginosa & $1(3.33)$ & - & & Pseudomonas spp (10\%) \\
\hline \multirow[t]{2}{*}{8} & Pseudomonas flourescens & $1(3.33)$ & - & & \\
\hline & Pseudomonas gladiolli & & - & & \\
\hline 9 & (Burkholderia gladiolli) & $1(3.33)$ & & & \\
\hline 10 & Kluyvera ascorbata & $1(3.33)$ & - & & \\
\hline 11 & Staphylococcus auerus & $2(6,67)$ & + & $46.67 \%$ & Staphylococcus spp \\
\hline 12 & Staphylococcus haemolyticus & $1(3.33)$ & + & & $(16.67 \%)$ \\
\hline 13 & Staphylococcus sciuri & $1(3.33)$ & + & & \\
\hline 14 & Staphylococcus schleiferi & $1(3.33)$ & + & & \\
\hline 15 & Streptococcus agalactiae & $3(10,0)$ & + & & Streptococcus spp (16.67\%) \\
\hline 16 & Streptococcus pyogenes & $1(3.33)$ & + & & \\
\hline 17 & Streptococcus porcinus & $1(3.33)$ & + & & \\
\hline 18 & Kytococcus sedentarius & $2(6,67)$ & + & & \\
\hline 19 & Kocuria kristiae & $1(3.33)$ & + & & \\
\hline \multirow[t]{2}{*}{20} & Kokus gram positif & $1(3,33)$ & + & & \\
\hline & TOTAL & $30(100)$ & & & \\
\hline
\end{tabular}


Table 2. Sensitivity Profile germs to antibiotics period August 2014- March 2015

\begin{tabular}{|c|c|c|c|c|c|c|c|}
\hline \multirow[b]{3}{*}{ Class of Antibiotics } & \multirow[b]{3}{*}{ Antibiotics } & \multicolumn{6}{|c|}{$\%$ Antibiotics Sensitivity } \\
\hline & & \multicolumn{3}{|c|}{ Gram Negatif } & \multicolumn{3}{|c|}{ Gram Positif } \\
\hline & & $\begin{array}{l}\text { E. coli } \\
(n=4)\end{array}$ & $\begin{array}{c}\text { Kleb. oxytoca } \\
(n=3)\end{array}$ & $\begin{array}{c}\text { Citro. } \\
\text { freundii } \\
(n=2)\end{array}$ & $\begin{array}{c}\text { Strep. } \\
\text { Agalactiae } \\
(n=3)\end{array}$ & $\begin{array}{c}\text { Staph. } \\
\text { Aureus } \\
(n=2)\end{array}$ & $\begin{array}{c}\text { Kytococ. } \\
\text { Sedent } \\
(n=2)\end{array}$ \\
\hline \multirow[t]{7}{*}{ Penisilin } & Penisilin & - & - & - & 0 & $0(1)$ & 100 \\
\hline & Ampisilin & 0 & 0 & 0 & 33,3 & $0(1)$ & 100 \\
\hline & Amoksisilin & - & - & - & - & - & - \\
\hline & Oksasilin & 0 & 0 & 0 & 0 & $0(1)$ & 50 \\
\hline & Amoks-klav & 0 & $0 / 33,3 i$ & 50 & - & $0(1)$ & - \\
\hline & Piperasilin & - & - & - & - & $100(1)$ & - \\
\hline & Metisilin & - & - & - & - & $0(1)$ & - \\
\hline \multirow[t]{7}{*}{ Sefalosporin } & Sefiksim & - & - & - & - & $0(1)$ & - \\
\hline & Sefadroksil & - & - & - & - & $0(1)$ & - \\
\hline & Sefotaksim & 0 & 33,3 & 100 & - & $0(1)$ & - \\
\hline & Sefazolin & 0 & 33,3 & $0 / 50 \mathrm{i}$ & - & - & - \\
\hline & Seftazidim & $0 / 25 \mathrm{i}$ & 33,3 & 100 & - & $100(1)$ & - \\
\hline & Seftriakson & 0 & 33,3 & 100 & $0(1)$ & - & $0(1)$ \\
\hline & Sefpirom & - & - & - & - & $100(1)$ & - \\
\hline \multirow[t]{2}{*}{ Karbapenem } & Imipenem & - & - & - & - & $100(1)$ & - \\
\hline & Meropenem & $0 / 25 \mathrm{i}$ & $0 / 66,7 \mathrm{i}$ & $0(1)$ & - & $100(1)$ & - \\
\hline Monobaktam & Aztreonam & 0 & 33,3 & $50 \mathrm{~s} / 50 \mathrm{i}$ & - & - & - \\
\hline Glikopeptida & Vankomisin & $100(1)$ & - & - & 66,7 & $100(1)$ & 50 \\
\hline Tetrasiklin & Tetrasiklin & 0 & 33,3 & 0 & 0 & 0 & 100 \\
\hline \multirow[t]{2}{*}{ Makrolida } & Eritromisin & - & - & - & 66,7 & $0(1)$ & 100 \\
\hline & Fosfomisin & - & - & - & - & $100(1)$ & - \\
\hline Fenikol & Kloramfenikol & - & - & - & - & $0(1)$ & - \\
\hline \multirow[t]{5}{*}{ Aminoglikosida } & Amikasin & 75 & $66,7 \mathrm{~s} / 33,3 \mathrm{i}$ & 100 & 100 & 100 & 100 \\
\hline & Dibekasin & - & - & - & - & $100(1)$ & - \\
\hline & Gentamisin & 75 & 33,3 & 100 & $66,7 \mathrm{~s} / 33,3 \mathrm{i}$ & 0 & 100 \\
\hline & Streptomisin & - & - & - & - & - & - \\
\hline & Tobramisin & 50 & 33,3 & 50 & 100 & $100(1)$ & 100 \\
\hline Folic inhibitor & TMP-SMZ & 0 & 33,3 & 50 & 66,7 & 0 & 50 \\
\hline \multirow[t]{4}{*}{ Quinolon } & Siprofloksasin & 0 & 33,3 & 50 & $66,7 \mathrm{~s} / 33,3 \mathrm{i}$ & 50 & 100 \\
\hline & Levofloksasin & 0 & 66,7 & 50 & $66,7 \mathrm{~s} / 33,3 \mathrm{i}$ & 50 & 100 \\
\hline & Ofloksasin & - & - & - & - & $100(1)$ & - \\
\hline & Norfloksasin & - & - & - & - & $100(1)$ & - \\
\hline
\end{tabular}

\begin{tabular}{|c|c|}
\hline \multicolumn{2}{|l|}{ Information } \\
\hline & Sensitivity $>60 \% \quad$ : recommended \\
\hline & Sensitivity $30-60 \%$ : considered \\
\hline & Sensitivity<30\% $:$ not recommended \\
\hline (number) & Bacterial number in senstivity test \\
\hline $\mathrm{s} / \mathrm{i}$ & Sensitive/intermediary \\
\hline
\end{tabular}

\section{Prospective Data}

For prospective data of 13 patients obtained the inclusion of 28 patients with diabetic foot, with 14 cultures and 14 isolates germs infecting.

\section{Patient demographics}

Table 3 shows the demographic data of patients diabetic foot patients who met the inclusion criteria which include gender of the patient, age, severity of disease, and the patient's status. Number 8 female patients $(61.54$ $\%)$, and men's number $5(34.46 \%)$. Patients age 50-60 years dominated as much 8orang $(61.54 \%)$, while the rate of infection of patients are mostly severe infections
(10 cases; $76.93 \%)$. Status patients mostly BPJS (9; $69.23 \%)$.

Table 3. Profile of patients with a diagnosis of diabetic foot infection period late March-early August 2015

\begin{tabular}{rlcc}
\hline \multicolumn{2}{l}{ Demography of Patient } & $\mathrm{N}$ & Percentage \\
\hline Sex & & \\
$-\quad$ Male & 5 & $38.46 \%$ \\
$-\quad$ Female & 8 & $61.54 \%$ \\
Age & & \\
$-\quad<50$ years old & $1(\mathrm{P})$ & $7.69 \%$ \\
$-\quad 50-60$ years old & $8(3 \mathrm{P}, 5 \mathrm{~L})$ & $61.54 \%$ \\
$-\quad>60$ years & $4(\mathrm{P})$ & $30.77 \%$ \\
Infection Severity & & \\
- Mild & - & $0 \%$ \\
$-\quad$ Moderate & 3 & $23.07 \%$ \\
\hline
\end{tabular}




\begin{tabular}{ccc}
\hline$-\quad$ Severe & 10 & $76.93 \%$ \\
Status of patient & & \\
$-\quad$ BPJS & 9 & $69.23 \%$ \\
$-\quad$ SPM & 1 & $7.69 \%$ \\
$-\quad$ Others & 3 & $23.08 \%$ \\
\hline
\end{tabular}

* Percentage is calculated based on the total number of patients that 13 patients

\section{Profile Germs on Diabetic Foot Infection}

Table 4 shows the types of bacteria that cause infections in the diabetic foot hospital inpatient Mardi Waluyo period end of March - beginning of August 2015. Of the 13 patients obtained 14 results hulkur pus which produces 14 isolates of bacteria with prevalence of gram-negative bacteria as much as $42.86 \%$ and grampositive as much as $57.14 \%$. Most gram-negative types are Klebsiella oxsytoca (28.57\%), and most gram positive Staphylococcus auerus (35.71\%).

Table 4. Type of germs infecting End of period March 2015 - Beginning August 2015

\begin{tabular}{rlccc}
\hline No & Type og Germs & $\mathrm{N}(\%)$ & Gram + / & Note \\
\hline 1 & Klebsiella oxsytoca & $4(28.57)$ & - & Gram (-) \\
2 & Providencia stuartii & $1(7.14)$ & - & $42.86 \%$ \\
3 & Morganella morganii & $1(7.14)$ & - & \\
4 & Staphylococcus auerus & $5(35.71)$ & + & Gram (+) \\
5 & Staphylococcus saccharolyticus & $1(7.14)$ & + & $57.14 \%$ \\
6 & Streptococcus agalactiae & $1(7.14)$ & + & \\
7 & Streptococcus $\beta$ haemolyticus & $1(7.14)$ & + & $100 \%$ \\
\hline \multicolumn{7}{c}{ Total } & $14(100)$ & & \\
\hline
\end{tabular}

\section{Sensitivity Profile Germs on the Diabetic Foot Infection Antibiotics}

Table 5. Sensitivity Profile germs to antibiotics Period End of March - Beginning August 2015

\begin{tabular}{|c|c|c|c|c|c|c|c|}
\hline \multirow[b]{3}{*}{ Class of Antibiotics } & \multirow[b]{3}{*}{ Antibiotics } & \multicolumn{6}{|c|}{$\%$ Antibiotics Sensitivity } \\
\hline & & \multicolumn{3}{|c|}{ Gram Negative } & \multicolumn{3}{|c|}{ Gram Positive } \\
\hline & & $\begin{array}{c}\text { Kleb. } \\
\text { oxytoca } \\
(n=4)\end{array}$ & $\begin{array}{c}\text { Provid. } \\
\text { Stuartii } \\
(n=1)\end{array}$ & $\begin{array}{c}\text { Morg. } \\
\text { morganii } \\
(n=1)\end{array}$ & $\begin{array}{l}\text { Strtococc. } \\
\operatorname{spp}(n=2)\end{array}$ & $\begin{array}{c}\text { Staph. } \\
\text { Aureus } \\
(n=5)\end{array}$ & $\begin{array}{c}\text { S.saccharo } \\
\text { lyticus } \\
(n=1)\end{array}$ \\
\hline \multirow[t]{7}{*}{ Penisilin } & Penisilin & - & - & - & $0(1)$ & 40 & 0 \\
\hline & Ampisilin & 0 & 100 & 0 & $100(1)$ & 20 & 0 \\
\hline & Amoksisilin & - & - & - & & - & - \\
\hline & Oksasilin & 0 & 0 & 0 & $0(1)$ & 0 & 0 \\
\hline & Amoks-klav & $25 \mathrm{~s} / 50 \mathrm{i}$ & 100 & 0 & $100(1)$ & - & - \\
\hline & Piperasilin & - & - & - & $100(1)$ & - & - \\
\hline & Metisilin & - & - & - & - & - & - \\
\hline \multirow[t]{7}{*}{ Sefalosporin } & Sefiksim & - & - & - & $100(1)$ & - & - \\
\hline & Sefadroksil & - & - & - & $0(1)$ & - & - \\
\hline & Sefotaksim & 75 & 100 & 100 & $100(1)$ & - & - \\
\hline & Sefazolin & 50 & - & 0 & - & - & - \\
\hline & Seftazidim & 75 & $\mathrm{i}$ & 100 & $100(1)$ & - & - \\
\hline & Seftriakson & 75 & 100 & 100 & - & - & - \\
\hline & Sefpirom & - & - & - & $100(1)$ & - & - \\
\hline \multirow[t]{2}{*}{ Karbapenem } & Imipenem & - & - & - & $100(1)$ & - & - \\
\hline & Meropenem & $50 \mathrm{~s} / 50 \mathrm{i}$ & 100 & $\mathrm{I}$ & & - & - \\
\hline Monobaktam & Aztreonam & 75 & 100 & 100 & & - & - \\
\hline Glikopeptida & Vankomisin & - & - & - & $100(1)$ & 100 & 100 \\
\hline Tetrasiklin & Tetrasiklin & $25 \mathrm{i}$ & 0 & 0 & 50 & 40 & 0 \\
\hline \multirow[t]{2}{*}{ Makrolida } & Eritromisin & - & - & - & $100(1)$ & $60 \mathrm{~s} / 20 \mathrm{i}$ & 0 \\
\hline & Fosfomisin & - & - & - & $100(1)$ & - & - \\
\hline Linkosamid & Klindamisin & - & - & - & $0(1)$ & - & - \\
\hline Fenikol & Kloramfenikol & - & - & - & $100(1)$ & - & - \\
\hline \multirow[t]{5}{*}{ Aminoglikosida } & Amikasin & 100 & 100 & 100 & $50 \mathrm{~s} / 50 \mathrm{i}$ & 80 & 0 \\
\hline & Dibekasin & - & - & - & $100(1)$ & - & - \\
\hline & Gentamisin & 75 & 100 & 100 & 50 & 100 & 0 \\
\hline & Streptomisin & - & - & - & $0(1)$ & - & - \\
\hline & Tobramisin & $75 \mathrm{~s} / 25 \mathrm{i}$ & 100 & $\mathrm{i}$ & $\mathrm{i}$ & 80 & 0 \\
\hline Folic inhibitor & TMP-SMZ & 25 & 100 & 0 & 50 & 100 & 0 \\
\hline \multirow[t]{4}{*}{ Quinolon } & Siprofloksasin & $25 \mathrm{~s} / 25 \mathrm{i}$ & 100 & 100 & $50 \mathrm{~s} / 50 \mathrm{i}$ & $60 \mathrm{~s} / 20 \mathrm{i}$ & 0 \\
\hline & Levofloksasin & 50 & 100 & 100 & 100 & 80 & 0 \\
\hline & Ofloksasin & - & - & - & $100(1)$ & - & - \\
\hline & Norfloksasin & - & - & - & $100(1)$ & - & - \\
\hline
\end{tabular}




\begin{tabular}{|c|l|}
\hline & Sensitivity $>60 \% \quad$ : recommended \\
\hline & Sensitivity $30-60 \%:$ considered \\
\hline & Sensitivity $<30 \% \quad$ : not recommended \\
\hline (number) & Bacterial number in sensitivity tyest \\
\hline s/i & Sensitive/intermediary \\
\hline
\end{tabular}

\section{Use of Antibiotics Profile in Patients with Diabetic Foot and the Qualitative Analysis}

The number of antibiotic therapy in 13 patients are as many as 14 kinds of antibiotics, where the patient can get more than one kind of regimentation of antibiotics with different doses and intervals. Originally a single administration of antibiotics can then for some reason there are additions or replacements both in terms of type, dose and administration interval so that the total number is 50 Award. Table 6 shows the suitability of dose and interval of antibiotics for the treatment of diabetic foot infection between guides guideline with reality given to the patient (by type). Rate the quality of the use of antibiotics in diabetic foot patients by category Gyssens performed in hospitals Mardi Waluyo Blitar City during the period of late March - early August 2015 having previously performed a retrospective data collection period August 2014 March 2015 who received antibiotic therapy, both empirical and definitive. The quality of antibiotic use were analyzed using flow Gyssens are divided into categories 0 to VI. From the analysis of the use of antibiotics with the highest use of antibiotics Gyssens method is more effective in $52 \%$ (category IV A); not appropriate intervals as much as $26 \%$ (category II B); teepat no dose by $14 \%$ (category IIA). Complete data analysis results with the use of antibiotics Gyssen method can be seen in Table 7.

Table 6. Interval Dose Antibiotic for Treatment Diabetic Foot (by type)

\begin{tabular}{|c|c|c|c|c|}
\hline AntibiotiCS & Antibiotic doses (literature) & Dose in patients & Frequency* & Note \\
\hline \multirow[t]{4}{*}{ Seftriakson } & 1-2g /d, iv (Lipsky,2012) & 2x1g/d,iv (empirical) & 2 & Appropriate \\
\hline & & $2 \times 1 \mathrm{~g} / \mathrm{d}$,iv (definitive) & 1 & Appropriate \\
\hline & & $2 \times 2 \mathrm{~g} / \mathrm{d}, \mathrm{iv}$ (empirical) & 3 & overdose \\
\hline & & $2 \times 2 \mathrm{~g} / \mathrm{d}$,iv (definitive) & 1 & overdose \\
\hline \multirow[t]{3}{*}{ Sefoperazon } & No data on guidelines of diabetic foot & 2x1g/d,iv (empirical) & 3 & Appropriate usual dose \\
\hline & infection (usual dose:2-4g/d,iv- 2x / 12j) & 3x1g/d,iv (empirical) & 6 & $\begin{array}{l}\text { Appropriate usual dose, } \\
\text { interval not appropriate }\end{array}$ \\
\hline & & 2x1g/d,iv (definitive) & 1 & Appropriate usual dose \\
\hline Sefotaksim & $\begin{array}{l}200 \mathrm{mg} / \mathrm{kg} / \mathrm{d}-\mathrm{iv} / 4-6 \mathrm{hr} \text { Enterobacteriaceae } \\
\text { (Bernard,2006) }\end{array}$ & 3x1g/d,iv (empirical) & 2 & $\begin{array}{l}\text { Not Appropriate dose } \\
\text { \&interval (underdose) }\end{array}$ \\
\hline \multirow[t]{3}{*}{ Metronidazol } & 3x500mg, iv/oral (Chahine, 2013) & 3x500mg, iv(empirical) & 5 & Appropriate \\
\hline & & $2 \times 500 \mathrm{mg}$, iv(empirical) & 1 & underdose \\
\hline & & 2x500mg,iv(definitive) & 1 & underdose \\
\hline Siprofloksasin & 2x400mg,iv-each 12hr (Chahine,2013) & 2x400mg,iv(empirical) & 5 & Appropriate \\
\hline \multirow[t]{2}{*}{ Levofloksasin } & $1 \times 750 \mathrm{mg} / \mathrm{d}$, iv/oral (Chahine,2013) or & 1x 500mg, iv (definitive) & 4 & \\
\hline & $1 \times 500 \mathrm{mg} / \mathrm{d}$ iv/oral \pm klindamisin & & & Appropriate \\
\hline \multirow[t]{2}{*}{ Gentamisin } & $\begin{array}{l}4 \mathrm{mg} / \mathrm{kg} / \mathrm{d}, \text { each } 24 \mathrm{hr} \\
\text { (combination)(Bernard,2006); }\end{array}$ & 2x80mg,iv(empirical) & 3 & $\begin{array}{l}\text { Appropriate dose, safer } \\
\text { each } 24 \mathrm{hr}\end{array}$ \\
\hline & conventional: $1-2,5 \mathrm{mg} / \mathrm{kg}$, each $8-12 \mathrm{hr}$. & 2x80mg,iv(definitive) & 2 & $\begin{array}{l}\text { Appropriate dose, safer } \\
\text { each } 24 \mathrm{hr}\end{array}$ \\
\hline Meropenem & $3 \times 1 \mathrm{~g}$, iv -each $8 \mathrm{hr}$ (Chahine, 2013) & $3 \times 1 g$, iv (definitive) & 2 & Appropriate \\
\hline \multirow[t]{2}{*}{$\begin{array}{l}\text { Pelastin/imipenem - } \\
\text { silastatin }\end{array}$} & $1-2 \mathrm{~g} / \mathrm{d}, \mathrm{iv}, 3-4 \mathrm{x}$ dose $(500 \mathrm{mg} \mathrm{iv} / 6 \mathrm{hr})$ & 2x1g,iv (empirical) & 1 & $\begin{array}{l}\text { Dose/d Appropriate, } \\
\text { interval tdk Appropriate }\end{array}$ \\
\hline & & 2x1g,iv (definitive) & 1 & $\begin{array}{l}\text { Dose/d Appropriate, } \\
\text { interval not appropriate }\end{array}$ \\
\hline Amikasin & $\begin{array}{l}\text { No data on guidelines of diabetic foot } \\
\text { infection (usual dose: } 5-7,5 \mathrm{mg} / \mathrm{kg} / \mathrm{dose} \text { - } \\
\text { each } 8 \mathrm{hr} \text { ) }\end{array}$ & 3x250mg,iv (definitive) & 1 & Appropriate \\
\hline \multirow[t]{2}{*}{ Tetrasiklin } & $\begin{array}{l}\text { No data on guidelines of diabetic foot } \\
\text { infection (usual dose: dws } 4 \times 250-500 \mathrm{mg}\end{array}$ & $\begin{array}{l}3 \times 500 \mathrm{mg} \text {, oral (definitive)- } \\
\text { discharge }\end{array}$ & 1 & Interval notAppropriate \\
\hline & oral) & $\begin{array}{l}4 \times 500 \mathrm{mg}, \text { oral (definitive)- } \\
\text { discharge }\end{array}$ & 1 & Appropriate \\
\hline Ampisillin & $\begin{array}{l}\text { Ampi-sulbaktam is suggested } 3 \mathrm{~g}, \mathrm{iv} / 6 \mathrm{hr} \\
\text { (ampisillin usual dose: } 4 \times 250- \\
1000 \mathrm{mg}, \text { iv/im each } 6 \mathrm{hr}\end{array}$ & $3 \times 1 g$, iv (definitive) & 1 & $\begin{array}{l}\text { Appropriate dose, interval } \\
\text { Not Appropriate }\end{array}$ \\
\hline Zibac/ Seftazidim & $\begin{array}{l}\text { 2g-iv, each 8-12hr (Chahine, 2013), } \\
\text { DIH: 500mg-1gr, each } 8 \mathrm{hr}\end{array}$ & 3x1g,iv (empirical) & 1 & Appropriate \\
\hline
\end{tabular}




\begin{tabular}{|c|c|c|c|c|}
\hline Vancep & $\begin{array}{l}30 \mathrm{mg} / \mathrm{kg} \text {,iv -2x/d (Bader, 2008) or } \\
15-20 \mathrm{mg} / \mathrm{kg}, \text { iv - each 8- } \\
12 \mathrm{hr}(\text { Chahine,2013) }\end{array}$ & $\begin{array}{l}\text { 4x500mg, syring pump } \\
\text { (definitive) }\end{array}$ & 1 & Interval not appropriate \\
\hline
\end{tabular}

Table 7. Results of analysis of the use of antibiotics with Qualitative Methods

\begin{tabular}{clcc}
\hline Categories & \multicolumn{1}{c}{ Evaluation } & Total & Percentage \\
\hline O & Antibiotic use appropriate/wise & 31 & $62 \%$ \\
I & Antibiotic use not on time & 1 & $2 \%$ \\
II A & Antibiotic use not appropriate in dose & 7 & $14 \%$ \\
II B & Antibiotic use not appropriate in admnistration interval & 13 & $26 \%$ \\
II C & Antibiotic use not appropriate in admnistration route & - & $10 \%$ \\
III A & Antibiotic use too long & 5 & - \\
III B & Antibiotic use too short & - & $52 \%$ \\
IV A & Other more effective antibiotics are present & 26 & $6 \%$ \\
IV B & Other less toxic/safer antibiotics are present & 3 & $6 \%$ \\
IV C & Other less costly antibiotics are present & 3 & $8 \%$ \\
IV D & Other narrower spectrum antibiotics are present & 4 & - \\
V & No indication of antibiotic use & - & - \\
VI & Medical record data not complete dan not evaluable & - &
\end{tabular}

\section{DISCUSSION}

Of the 30 isolates germs infecting (retrospective data) obtained patterns of gram-negative bacteria dominated by as much as $53.33 \% 13.33 \%$ E coli, and Klebsiella oxytoca $13.33 \%$, the rest is Enterobacter spp, Citrobacter spp, Pseudomonas spp well, and gram positive as much as $46.67 \%$ dominated by $16.67 \%$ of Staphylococcus spp, Streptococcus spp (16.67\%). As for prospective data from 14 isolates of gram-negative bacteria gained as much as $42.86 \%$ and gram positive as much as $57.14 \%$. Seen a shift in the percentage of gram negative and positive emerging, most types of gramnegative is Klebsiella oxytoca $(28.57 \%)$, and most gram positive Staphylococcus auerus (35.71\%). GPC especially Staphylococcus aureus and beta-hemolytic streptococci is the organism most often in patients with mild-moderate DFI, and patients who did not receive antibiotic therapy in the previous month. Patients with a history of chronic infection and have taken antibiotics tend to develop into a mixed infection between GPC and GNB with or without anaerobic organisms. The existence of obligate anaerobes associated with necrotic, gangrenous or ischemic tissue, and this is usually a chronic and severe infection (Chahine in 2013).

Antibiotic therapy can be as empirical and definitive treatment. The principle of election empiric antibiotics are: a) the spectrum of activity of antibiotics, b) the ability to penetrate the network good, c) take into account the patient (the severity of the infection, allergies, kidney disorders), d) the map data germs and patterns of antibiotic resistance local, and e ) security and ease of administration to patients (Lipsky, 2007, Frykberg 2002, Cunha 2010). There are various recom- mendations on the use of empiric antibiotics in diabetic foot infections based on the level of infection, such as: a) mild-moderate infections; given the oral fluoroquinolones or aminopenisillin (amoksisillin-cla-vulanate, sulbactam ampisillin), with alternative clindamycin or Bactrim; b) moderate to severe infections, given the combination of clindamycin-ciprofloxacin intravenously; ceftazidime-metronidazole. Another recommendation for patients who have not been treated or cephalosporin antibiotics are aminopenisillin 2nd generation/3rd, to which had been treated antibioika: 3rd generation cephalosporin/4th, or fluoroquinolones + clindamycin; c) a life-threatening infection: class of carbapenem or aminoglycoside + clindamycin or a cephalosporin 3rd generation/4th + glycopeptide/ linezolid or fluoroquinolones + metronidazole (Lipsky 2007, Pranoto 2010).

Meanwhile, according to Chahine (2013), an antibiotic that can be recommended are: a) Mild infections: amoksisillin-clavulanate, cephalexin, clindamycin, dikloksasillin, levofloxacin, doxycycline and cotrimoxazole, given orally; b) moderate to severe infections: ampisillin sulbactam, sefoksitin, ceftriaxone, ciprofloxacin + clindamycin, ertapenem, imipenem-silastatin, levofloxacin + clindamycin, meropenem, moxifloxacin, tigesiklin, daptomisin, linezolid, and vancomycin; administered intravenously and each with regard to the nature of germs infecting (Table 2.15) (Chahine in 2013).

In this study, empirical antibiotic that is widely used is ceftriaxone, sefoperazon, cefotaxime, ciprofloxacin, metronidazole and gentamicin, either alone or in combination use. Based on the antibiogram, the data 
sensitivity ceftriaxone and cefotaxime showed potentials $>60 \%$ (which means it can be recommended usage) only on bacteria Citrobacter freundii only, while for the bacteria Klebsiella oxytoca both have the potential between $30-60 \%$ (can be considered its use), but no data sensitivity to sefoperazon, while ciprofloxacin potentially deadly $>60 \%$ on Streptococcus agalactiae and Kytococcus sedentarius, and may be considered for the bacteria Klebsiella oxytoca, Citrobacter freundii, and Staphylococcus aureus (sensitivity between 30-60\%). Gentamicin potentially deadly $>60 \%$ of bacteria $E$. coli, Citrobacter freundii, Stretococcus agalactiae and Kytococcus sedentarius, and can be considered the Klebsiella bacteria oxsitoca (sensitivity between 30$60 \%$ ). For metronidazole more focused on anaerobic bacteria, which are often found in the condition of necrotic, gangrenous or ischemic tissue, and this is usually a chronic and severe infection. In this study, metronidazole is not determined its potential against anaerobic bacteria. Based on the research of diabetic foot wounds pus isolates $(n=120$ isolates $)$ in anaerobic test showed that metronidazole has a potential $99 \%$ against all anaerobic bacteria tested and have a low level of resistance compared with clindamycin (Syng et al, 2008).

Based on the results of data processing retrospektik antibiogram, antibiotic potential is huge against gram negative and positive is amikacin (> 60\%: against $E$. coli, Kelb. Oxytoca 75\%, 100\% against Citrobacter freundii), and between $33.3 \%$ against Pseudomonas spp; $100 \%$ of Streptococcus spp, Staphylococcus spp, and Kytococcus sedentarius; Gentamicin (> 60\%: against $E$. coli, Kelb. Oxytoca 75\%, 100\% against Citrobacter freundii), and $33.3 \%$ against Pseudomonas spp; $66.7 \%$ against Streptoccus agalactiae, $100 \%$ of the Kytococcus sedentarius, and $40 \%$ against Staphylococcus spp.Terlihat also the potential of ciprofloxacin against gram-positive: $66.7 \%$ against Streptoccus agalactiae, $100 \%$ of the Kytococcus sedentarius and between 30$60 \%$ of the Kelb , Oxytoca and Citrobacter freundii. Levofloxacin looks more potent than ciprofloxacin in Klebsiella bacteria oxsitoca prevalence $13.33 \%$ in the diabetic foot. While vancomycin and tobramycin is more potent against gram-positive.

In the last 4 months period looked patterns germs little change related types of bacteria that appears, with the most prevalent gram-negative is Klebsiella oxytoca $(28.57 \%)$, where sensitivity> $60 \%$ of the 3 rd generation cephalosporins (75\%) - the highest amikacin $100 \%$; between $30-60 \%$ of cefazolin, meropenem and levofloxacin; and most gram positive Staphylococcus auerus $(35.71 \%)$, where sensitivity> $60 \%$ to vancomycin (100\%), amikacin (80\%), gentamicin (100\%), tobramycin (80\%), cotrimoxazole (100\%), and levofloxacin $(80 \%)$; antara30-60\% sensitivity to tetracycline, erythromycin and ciprofloxacin.

Generally from antibiogram picture shows antibiotic may mengkafer gram negative and positive bacteria is amikacin, gentamicin, ciprofloxacin and levofloxacin. Generation cephalosporin astreonam 3And all the more potent against gram-negative, and vancomycin 100\% potent against gram-positive. However, to be recommended as empiric antibiotics in diabetic foot patients would have to consider other factors. The age of patients for diabetic foot are mostly 50-60 year in which the function of organs, especially the kidneys have a tendency to 17 times higher chance of developing chronic renal failure than in healthy people, must be very careful in the use of aminoglycoside antibiotic class which has the side effect of nephrotoxic, neurotoxic, superinfection (C.difficile infection) on long-term use of gentamicin, and neuromuscular blockade or respiratory paralysis on amikacin (Lacy 2009). Besides the potential for higher antibiotic against bacteria in vitro infecting not necessarily effective in vivo. Conditions of acute injuries/chronic as well as the severity of the infection is also an important consideration. Based on these things, and refers to the guidelineguideline concerning empirical treatment of diabetic foot infection, we would recommend the use of ciprofloxacin or levofloxacin as empiric antibiotics in diabetic foot patients in hospitals Mardi Waluyo of Blitar. When should use aminoglycoside class, of course, it must be ensured also normal kidney function and do monitoring side effects. To mengkafer anaerobes, klidamisin oral/metronidazole iv/orally may be combined with ciprofloxacin or levofloxacin (Bader 2008, Lipsky 2012, Chahine in 2013).

The use of antibiotics in addition to considering the results of the culture, to note the patient's response to infection. If the lesion injuries to the patients improved and patients respond to empiric therapy, the replacement of antibiotics may not be necessary, although the results of antibiotic sensitivity test found infecting bacteria that are resistant to antibiotics that have been used empirically (Lipsky 1999). The use of broad-spectrum empiric antibiotics is required at the start of therapy (for information infecting bacteria is not known), when the results of culture and sensitivity test are known, should be done with the replacement of narrow-spectrum antibiotics. Due to the use of broad-spectrum empiric too long will lead to a selective process in which the pressure will increase the population of resistant bacteria and can alter the body's normal flora (Southwick 2007).

The results of the qualitative analysis using the method Gyssens obtained the use of antibiotics is appropriate 
(category 0) as much as $62 \%$, the use of atibiotika not timely (category 1) as much as $2 \%$, not appropriate dose (category 2A) by 14\%; not appropriate interval Award (category 2B) were 26\%; antibiotics for too long (category $3 \mathrm{~A}$ ) as much as $10 \%$; there are other antibiotics that are more effective (class $4 \mathrm{~A}$ ) in $52 \%$, there are other safer antibiotics (category $4 \mathrm{~B}$ ) as $6 \%$; there are other antibiotics that spectrum is narrower (category 4C) as much as $8 \%$ and no use of antibiotics in the category V and VI category.

\section{CONCLUSION}

From this analysis can gyssen data showed that the use of antibiotics in diabetic foot patients in hospitals Maerdi Waluyo Blitar City is dominated by inaccuracy in the selection of antibiotics (some are more effective), and the inappropriateness of antibiotics interval. It is therefore expected with this study is encouraging clinicians to further improve the quality of antibiotic use based on the pattern of germs.

\section{REFERENCES}

Adibhatla, R. M., Hatcher, J. F., \& Larsen, E. C. (2005). CDP-choline significantly restores phosphatidylcholine levels by differentially affecting phospholipase A2 and CTP:phosphocholine cytidyltranscytidyltransferase after stroke. J. Biol. Chem , 10, 6718-6725.

Adibhatla, R., Hatcher, J., \& Dempsey, R. (2001). Effect of Citicoline on Phospholipid and Glutathione Levels in Transient Cerebral Ischemia. Stroke , 32: 2376-2381.

American Diabetes Association (ADA), 2008. Diagnosis and Classification of Diabetes Mellitus. Diabetes Care. Vol. 30, pp.42-47.

American Diabetes Association (ADA). 2012. Standards of Medical Care in Diabetes-2012. Diabetes Care, Vol. 35, Suppl.1.

Archer G.L. and Polk R.E., 2010. Treatment and Prophylaxis of Bacterial Infections. In Kasper D.L., Fauci A.S. Harrison's Infectious Disease, New York, The McGraw-Hill Companies, Inc., p. 354-374.

Azmi s,2000, Haemorologi pada gangguan darah perifer dalam kumpulan makalah symposium Diabetic Peripheral Vascular Diseases and its Management, 2000; 3-5.

Bader, M., 2008. Diabetic Foot Infection. American Family Physician, Vol.78, No. 1, p.71-79

Beckert Stefan, et.al., A New Wound Based Severity Score for Diabetic Foot Ulcers, 2004; p.435-437.
Bernard, Louis, 2006, Management of Diabetic Foot Infections, Clinical Practice Guideline, In Medecine et Maladies Infectieuses, 37(2007):14-25

Boulton AJ. The Diabetic Foot. Blackweel Publising, 2002.

Boulton, A., Bowling., F,. 2007. Diabetic Foot Ucers In: Pharmacotherapy of Diabetes: New Development, eds. Mogensen, CE, New York: Springer

Boyko. A Prospective Study of Risk factor For Diabetic Foot ulcer. The Seattle Diabetic Foot Study, Departement of Medicine of Washington, Seattle, USA, 1999.

Castellanos, M., Sobrino, T., \& Castillo, J. (2006). Evolving paradigms for neuroprotection: molecular identification of ischemic penumbra. Cerebrovasc Dis , Suppl 2: 71-9.

Chahine, Elias B, et.al., 2013, Diabetic Foot Infection: An Update on Treatment, US Pharm, 2013;38(4)23-26

Clark, David P, 2010, Molecular Biology, Elsevier Inc, London (British Library Cataloging-in-Publication Data), p. 597

Clinical and Laboratory Standards Institute, Performance Standards for Antimicrobial Susceptibility Testing, M100-S16 Vol.26, No. 3, CLSI, Villanova, Pa., 2007.

Cook,C.L., Johnson, J.T., Wade, W.E., 2008. Diabetes Mellitus. In: M.A. Chisholm-Burns, B.G. Wells, T.L. Scheinghammer, P.M. Malone, J.M. Kolesar, J.C. Rotschafer, J.T. Dipiro (Eds.). Pharmacotherapy Principle \& Practice. USA: McGraw-Hill Co.

Cunha B.A., 2009. Skin and Soft Tissue Infection in Diabetes . Available emedicine, Medscape.com

Cunha, Burke A, 2010, Antibiotic Essentials, 9th edition, Physicians Press

Dávalos, A., Alvarez Sabín, J., Castillo, J., Díez Tejedor, E., Ferro, J., Martínez Vila, E., et al. (2012). Citicoline in the Treatment of Acute Ischaemic Stroke: An International, Randomised, Multicenter, Placebocontrolled Study (ICTUS Trial). The Lancet , 380: 349-357.

Departemen Kesehatan RI. (2007). Riset Kesehatan Dasar. Jakarta: Departemen Kesehatan RI.

DiPiro, J. T., Talbert, R. L., Yee, G. C., Mazke, G. R., Wells, B. G., \& Posey, L. M. (2008). A Pharmacotherapy: Pathophysiologic Approach, 8th ed. New York: The McGraw Hills.

Dirnagl, U., Ladecola, C., \& Moskowitz, M. (1992). Pathobiology of ischemic stroke: an integrated view. Trends Neurosci , 22: 391-397.

Farooqui, A., Horrocks, L., \& Farooqui, T. (2005). Glycerophospholipids in brain: their metabolism, incorporation to membranes, functions, and involvement in neurological disorders. Chem. Phys. Lipids , 10: 6718-6725.

Fauci AS, Kasper DL, 2010, Harrison's Infectiuous Diseases, McGraw Hill Companies Inc., New York 
Frier, BM, Fisher, M 2007, Diabetes Mellitus, In Davidson's Principle and Practice of Medicine, eds Boon, NA, Colledge, NR, Walker, BR, 20th ed, Churchill Livingstone

Frykberb Robert G. Risk Factor, Pathogenesis and Management of Diabetic Foot Ulcers, Des Moines University, Iowa, 2002.

Frykberg, R.G., Zgonis, T., Armstrong, D.G., Driver, V.R., Giurini, G.M., Kravitz, S.R., Lansman, A.S., Lavery, L.A., Moore, J.C., Schuberth, J.M., Wukich, D.K., Andersen, C., Vanore, J.V., 2006. Diabetic Foot Disorders: A Clinical Practice Guideline (2006 revision). The Journal of Foot \& Ankle Surgery, Vol. 45, No. 5, S.2- S.66

Gadepalli R et.al., 2006, A Clinico Microbiological Study of Diabetic Foot Ulcer In An Indian Tertiary Care Hospital, Diabetes Care, 29:1727-1732

Gayle ER. Footwear used by individuals with diabetes and a history of foot ulcer Departments of Health Services, Joslin Diabetes Center at Swedish Medical Center, Seattle, 2002.

Gyssens I.C. 2005. Audits for Monitoring the Quality of Antimicrobial Prescriptions. In: Gould IM., van der Meer JWM., Antibiotic Policies: Theory and Practice. Kluwer Academic/Plenum Publishers New York.

Gyssens I.C., 2011. Antibiotic policy. J Antimicrob Ag, Elsevier 38S, 11-20

Heart and Stroke Foundation. (2003). Let's Talk About Stroke: An Information Guide for Survivors and Their Families. Ottawa: Heart and Stroke ca.

Hindler and Stelling, 2007. Analysis and Presentation of Cumulative Antibiograms: A New Consensus Guideline from the Clinical and Laboratory Standards Institute. Medical Microbiology, Clin Infect Dis 44:867-73

Hurtado, O., Moro, M. A., Cardenas, A., Sanchez, V., Tome, P. F., Leza, J. C., et al. (2005). Neuroprotection afforded by prior citicoline administration in experimental brain ischemia: effects on glutamate transport. Neurobiology of Disease , 18: 336-345.

James H. Jorgensen \& Mary Jane Ferrare, Antimicrobial Susseptibility Testing: A Review of Principles and Contemporary Practice, Medical Microbiology, CID, 2009:49

Jorgensen, M., \& Diemer, M. (1982). Selective neuron loss after cerebral ischemic in the rat: Possible role of transmitter glutamate. Acta Neuro Scand , 536-544.

Krupinski, J., Ferrer, I., Barrachina, M., Secades, J., Mercadal, J., \& Lozano, R. (2002). CDP-choline reduces pro-caspase and cleaved caspase-3 expression, nuclear DNA fragmentation, and specific PARPcleaved products of caspase activation following middle cerebralartery occlusion in the rat. Neuropharmacology , 42: 846-854.

Kumar \& Clark, 2009. Antimicrobial Chemotherapy In: Clinical medicine, sixth edition, p 34-5
Lacy, C.F., Armstrong, L.L., Goldman, M.P., Lance, L.L., 2009. Drug Information Handbook, 18th ed, USA: American Pharmacist Association

Lampiris H.W. and Maddix D.S., 2007. Clinical Use of Antimicrobial Agents. In Katzung B.G. Basic and Clinical Pharmacology 10th edition, New York, The McGraw-Hill Companies, Inc., p. 827-841

Levin ME, 2001. Pathogenesis and General Management of Foot lesions in the Diabetic Patiens. Dalam:Levin ME, editors, The Diabetic Foot, edisi 6, St Louis, The CV Mosby Company

Lipsky, B.A, et.al., 2012, Infectiuous Diseases Society of America Clinical Practice Guideline for The Diagnosis and Treatment of Diabetic Foot Infections (IDSA Guideline, 2012), Published by Oxford University Press, Clinical Infectiuous Disease; 54(12)132-173

Lipsky, B.A., Berendt, A.R., Deery, H.G., Embil, J.M., Joseph, W.S., Karchmer, A.W., LeFrock, J.L., Lew, D.P., Mader, J.T., Norden, C., Tan, J.S., 2004. IDSA GUIDELINES: Diagnosis and Treatment of Diabetic Foot Infections. Clinical Infectious Diseases, Vol. 39, p. $85-910$

Lo, E., Dalkara, T., \& Moskowitz, M. (2003). Mechanisms, challenges an opportunities in stroke. Nat Rev Neurosci , 4: 399-414.

Martinet, M., Fonlupt, P., \& Pacheco, H. (1979). Effects of cytidine-5' diphosphocholine on norepinephrine, dopamine and serotonin synthesis in various regions of the rat brain. Arch Int Pharmacodyn Ther , 239(1):52-61.

Martynov, M. Y., Boiko, A. N., Kamchatnov, P. R., Kabanov, A. A., Yasamanova, A. N., Shchukin, I. A., et al. (2013). Neuroprotective Therapy with Citicoline (Ceraxon) in Patients with Ischemic Stroke. Neuroscience and Behavioral Physiology , 43: 706711.

Misnadiarly. Diabetes Mellitus: Ulcer, Infeksi, Ganggren. Penerbit Populer Obor, Jakarta, 2006.

NHS, 2012, Diabetic Foot Problems - Inpatient Management, National institute for Health Clinical Excellence (NICE Clinical Guideline 119)

Novadian, Elvidawati, Shahab A: Pattern of Germ Diabetic Foot and Sensitivity to Antibiotic in RS Dr M, Hoesin in Konggres perhimpunan Penyakit Dalam XIII, Palembang, 2006, p. 159-160

Overgaard, K. (2014). The Effects of Citicoline on Acute Ischemic Stroke: A Review. Journal of Stroke and Cerebrovascular Diseases , vol 23: 1764-1769.

PERKENI. Konsensus Pengelolaan dan Pencegahan Diabetes Mellitus Tipe 2 diIndonesia, 2006.

Pract. Risk Factors of Diabetic Foot Ulcer a Case Control Study. Journal of Family Practise, USA, 2000.

Purba, J. M. (2011). Biomolekuler Stroke. In J. Misbach, Stroke: Aspek Diagnostik, Patofisiologi, 
Manajemen (pp. 41-56). Jakarta: Badan Penerbit FKUI.

Richard, C., \& Schauss, G. A. (2004). Therapeutic Applications of Citicoline for Stroke and Cognitive Dysfunction in the Elderly: A Review of the Literature. Alternative Medical Review , 9 (1): 17-31.

Richard,J.K, et.al, New Insights in Diabetic Foot Infection, Guideline for Clinical Practice, World Journal of Diabetes, 2011 feb 15; 2(2): 24-32

Riyanto B. Infeksi pada Kaki Diabetik. Dalam: Darmono, dkk, editors. Naskah Lengkap Diabetes Mellitus Ditinjau dari Berbagai Aspek Penyakit dalam dalam rangka Purna Tugas Prof Dr.dr.RJ Djokomoeljanto. Badan Penerbit Universitas Diponegoro Semarang, 2007. p.15-30.

Ryan KJ, Ray CG, 2004, An Itroduction to Infectiuous Diseases, Sherris Medical Microbiology, 4th edition, McGraw Hill, p.261-271

Sahota, P., \& Savitz, S. (2011). Investigational Therapies for Ischemic Stroke: Neuroprotection and Neurorecovery. Neurotherapeutics , 8: 434-451.

Sapico FL. Food Ulcer in Patients with Diabetes Mellitus, Journal of American Podiatric Medical Association, Vol 79, Issue 482-485, diakses tanggal 12 Januari 2007.

Schena, F.P., Gesualdo L., 2005. Pathogenetic Mechanisms of Diabetic Nephropathy. American Society of Nephrology. J Am Soc Nephrol 16: S30S33

Secades, J. (2002). CDP-choline: Updated pharmacological and clinical review. Methods Find. Exp. Clin. Pharmacol , 24: 1-56.

Shaw JE, et al. Global estimates of the prevalence of diabetes for 2010 and 2030, Diabetes Res Clin Pract. 2010 Jan; 87(1):4-14. Doi: 10.1016/j.diabres.2009.10.007. Epub 2009 Nov 6.

Stephen J. Cavaleri, et.al., 2005, Manual of Antimicrobial Susceptibility Testing, American Society for Microbiology

Subekti I. Neuropati Diabetik. Dalam: Aru W, dkk, editors, Ilmu Penyakit Dalam, Jilid III, Edisi keempat, Penerbit FK UI, Jakarta, 2006.

Tambunan M: Perawatan Kaki Diabetes dalam Penatalaksanaan Diabetes Mellitus Terpadu, Pusat Diabetes dan Lipid, Balai Penerbit FKUI, 2004, 293 298

Tjokroprawiro, A., Hendromartono, Sutjahjo, A., Pranoto, A., Murtiwi, S., Soebagjo, A.S., Wibisono,
S., 2007. Diabetes Mellitus In: Buku Ajar Ilmu Penyakit Dalam, eds. Tjokroprawiro, A, Setiawan, PB, Santoso, D, Soegiarto, G, Surabaya: Airlangga University Press.

Tortora G.J., Funke B.R., Case C.L., 2010. Microbiology an Introduction 10th edition, San Francisco, Pearson Education, Inc., p. 553-581

Triplitt, C.L., Reasner, C.A., Isley, W.L., 2008. Diabetes Mellitus. In: Dipiro, J. T., Talbert, R. L., Yee, G. C., Matzke, G. R., Wells, B. G., Posey, L. M., Pharmacotherapy: A Pathophisiologic Approach, Edisi ke-7, New York: The McGraw-Hill Companies, Inc.

Van Der Meer and Gyssen I.C., 2001. Quality of Antimicrobial Drug Prescription in Hospital, Eur Soc of Clin Microb and Infect Dis, p. 12-15

Wagner, F.W., 1983. Algorithms of diabetic foot care. In: The Diabetic Foot, 2nd Edition. Eds Levin ME, O’Neal LW. St.Louis: Mosby

Wahlgren, N. (1997). Neuroprotectants in Late Clinical Development - A Status Report. In Cerebrovascular Disease. 7 (Suppl 2): 13-17.

Warach, S., Pettigrew, L. C., Dashe, J. F., Pullicino, P., Lefkowitz, D. M., Sabounjian, L., et al. (2000). Effect of citicoline on ischemic lesions as measured by diffusion-weighted magnetic resonance imaging. Citicoline 010 Investigators. Ann Neurol , 48 (5): 71322.

Waspadji S. Kaki Diabetes. Dalam: Aru W, dkk, editors, Ilmu Penyakit Dalam, Jilid III, Edisi keempat, Penerbit FK UI, Jakarta 2006.

Waspadji S. Komplikasi kronik Diabetes: Mekanisme Terjadinya, Diagnosis dan Strategi pengelolaan. Dalam: Aru W, dkk, editors, Ilmu Penyakit Dalam,Jilid III, Edisi keempat, Penerbit FK UI, Jakarta, 2006.

WHO. Prevention of Diabetes Mellitus. Technical Report Series 844, Geneva, 2000.

Wild S, Roglic G, Green A, Secree R, King H, Glonal prevalence of diabetes: estimates for the year 2000 and projection for 2030. Diabetes Care 2004 May;27(5):1047-53.

William C. The Diabetic Foot, In ( Ellenberg, Rifkin's, eds), Diabetes Mellitus, Sixth Edision, USA, 2003.

Worl Health Organization. (2006). Neurological disorder: public health challenges. Geneva, WHO Press, World Health Organization. 